Slavica

bruxellensia

\section{Slavica bruxellensia}

Revue polyphonique de littérature, culture et histoire

slaves

5 | 2010

Après 1989

\title{
Books that shook Russia
}

Sur La Russie en 1839 du marquis de Custine

\section{Emmanuel Waegemans}

Traducteur : Katia Vandenborre

\section{OpenEdition}

Journals

Édition électronique

URL : http://journals.openedition.org/slavica/306

DOI : $10.4000 /$ slavica.306

ISSN : 2034-6395

Éditeur

Université libre de Bruxelles - ULB

Édition imprimée

Pagination : 81-85

ISSN : 2031-7654

\section{Référence électronique}

Emmanuel Waegemans, «Books that shook Russia », Slavica bruxellensia [En ligne], 5 | 2010, mis en ligne le 15 février 2010, consulté le 21 avril 2019. URL : http://journals.openedition.org/slavica/306 ; DOI : 10.4000/slavica.306

Ce document a été généré automatiquement le 21 avril 2019

\section{(c) (i) $\odot$}

Les contenus de Slavica bruxellensia sont mis à disposition selon les termes de la Licence Creative Commons Attribution - Pas d'Utilisation Commerciale - Pas de Modification 3.0 France. 


\title{
Books that shook Russia
}

Sur La Russie en 1839 du marquis de Custine

\author{
Emmanuel Waegemans
}

Traduction : Katia Vandenborre

\section{NOTE DE L'ÉDITEUR}

La langue originelle de cet article est le néerlandais

1 La Russie a été redécouverte par les Occidentaux au XVI ${ }^{e}$ siècle, après la domination mongole et l'établissement de la principauté de Moscou. Le premier livre sur la Russie sorti à l'époque en Occident qualifiait le pays de «rude and barbarous kingdom $»^{1} \ldots$ L'auteur anglais se trouvait dans le pays sous le règne d'Ivan le Terrible (pas vraiment le tsar le plus sympathique) et y a été le témoin de la guerre sanglante qu'Ivan IV mena contre les boyards. Ce point de vue est devenu un cliché de la Russie : cruelle et barbare. (Qu'aurait écrit un voyageur italien sur les Pays-Bas du sud s'il s'était retrouvé dans la «Furie espagnole»?) Les vastes contacts entre les marchands anglais et néerlandais et les diplomates occidentaux aux $\mathrm{XVI}^{\mathrm{e}}$ et $\mathrm{XVII}{ }^{\mathrm{e}}$ siècles sont à l'origine de la publication de nombreux livres qui ont été lus pendant des décennies, voire parfois même pendant des siècles, par les voyageurs sur le point de partir en Russie. Les livres les plus lus étaient : Rerum Moscoviticarum Commentarii (1549) de Sigismund von Herberstein, Moscovia (1586) de Antonio Possevini, Orientalische Reise (Voyage oriental, 1647) d'Adam Olearius, Histoire des Guerres de la Moscovie (début du XVII e siècle) d'Isaac Massa, Noord en Oost Tartarye (Tatarie du nord et de l'est, 1692) de Nicolaes Witsen. Si on les lit attentivement et dans l'ordre chronologique, il ressort que la plupart des voyageurs empruntent à l'un et à l'autre et que donc les jugements, voire les préjugés, d'un livre finissent dans l'autre.

2 Les réformes déchaînées de Pierre le Grand et le brillant gouvernement de Catherine II renforcèrent encore l'intérêt des Occidentaux pour le sphinx de l'Est. Mais le XVIII siècle n'a fourni aucun livre sur la Russie qui ait résisté à l'épreuve du temps (sauf peut-être 
Viaggi di Russia de Francesco Algarotti, qui a visité le pays en 1739 et a lancé la célèbre formule "Saint-Pétersbourg est la fenêtre par laquelle la Russie regarde l'Occident »²).

C'est aussi le cas de La Russie en 1839, le livre du Français Astolphe de Custine paru en 1843. Son père et son grand-père étaient des soldats qui avaient sympathisé avec les révolutions (américaine et française). Ils allèrent tous deux se faire trancher la gorge par Robespierre. Le marquis de Custine a composé plusieurs œuvres littéraires, mais elles sont à peu près passées inaperçues et nous n'aurions sans doute jamais entendu parler de lui s'il n'était pas allé en Russie et n'avait pas écrit un livre à ce sujet. Le sort de son père et de son grand-père ont fait du jeune marquis un ardent partisan de la monarchie. Il partit dans la Russie de Nicolas I ${ }^{\text {er }}$ pour s'assurer lui-même sur place de la grande vertu d'une monarchie autoritaire réactionnaire. Il en est revenu républicain. Le marquis passa en tout deux mois en Russie (Saint-Pétersbourg, Moscou, Iaroslav, Nijni-Novgorod, Vladimir). Le livre qu'il écrivit sur le pays parut quatre ans après son séjour et connut un succès immédiat. Le livre fut traduit dans toute l'Europe et, déjà en 1854, deux cent mille exemplaires avaient été vendus. En Belgique, même une édition pirate vit le jour. En matière de succès, le gouvernement russe en fut moins heureux. Le marquis de Custine avait été reçu partout dans la capitale avec tous les égards, peut-être à cause de sa réputation royaliste, et pourtant il ridiculisait le pays. En France de surcroît.

Si l'on met de côté les aventures personnelles et les anecdotes, le livre est toujours porteur d'un message stupéfiant:

La Russie est un pays misérable. Soixante millions de personnes sont condamnées à vivre en Russie.

Les Russes n'ont pas le sens de la justice.

7 L'état de siège est une situation normale en Russie : tout est interdit, sauf ce qui est explicitement autorisé et encore, en pratique, c'est interdit.

8 Les Russes et le gouvernement russe en particulier sont très sensibles à ce que les étrangers disent à leur sujet et à ce qu'ils rapportent dans la presse occidentale.

Quand en Russie un dignitaire est congédié ou qu'un tsar disparaît (à la suite d'un meurtre par exemple), il devient tout à coup plus personne et plus personne ne parle (ou n'ose parler) de lui.

La Russie est isolée du monde extérieur, la libre communication avec l'Occident ne pourrait survivre au régime.

Un étranger ne peut jamais faire le tour de la ville seul, des guides officiels sont toujours accrochés à lui.

Vous ne saurez jamais ce que vous voulez vraiment savoir.

Dans la presse, on ne parle pas des choses négatives.

Si une révolution éclate en Russie, elle se passe dans le sang.

Les autorités mentent effrontément aux étrangers.

La Russie a beaucoup à cacher (il n'y a pas de liberté de la presse) : "Qu'on accorde pendant vingt-quatre heures la liberté de la presse à la Russie, ce que vous apprendrez vous fera reculer $\mathrm{d}^{\prime}$ horreur. $»^{3}$

Les Russes sont réprimés, mais ils en sont reconnaissants.

18 Il est clair que le gouvernement de Nicolas I ${ }^{\text {er }}$ n'a pas été établi en vue d'accomplir cette mission destructrice. Le règne de Nicolas a commencé avec la désastreuse tentative de 
coup d'État des Décembristes le 14 décembre 1825 (préparé à la hâte, comme c'est souvent le cas dans l'histoire russe). Cette tentative audacieuse d'introduire une monarchie constitutionnelle a convaincu Nicolas $\mathrm{I}^{\mathrm{er}}$ qu'il fallait régner sur le pays d'une main de fer et contenir toutes les idées progressistes et révolutionnaires (comme il avait d'ailleurs été décidé par la Sainte Alliance entre la Prusse, l'Autriche et la Russie). Nicolas Ir était bien conscient du caractère insoutenable du servage et il aurait voulu y remédier, mais ses conseillers conservateurs le lui déconseillèrent vivement. Quand Pëtr Čaadaev publia sa célèbre Lettre philosophique (1836), ce fut le début de la fin. Un des hommes les plus brillants de son temps (ami de Puškin) avait mis l'histoire et la culture de la Russie à la poubelle, considérant que le pays devait chercher une connexion dans le catholicisme afin qu'il s'épanouisse comme une nation dynamique. L'homme fut déclaré fou. Dans les années 1840, d'après le témoignage de ses contemporains, Nicolas $\mathrm{I}^{\mathrm{er}}$ imposa « un silence du cimetière »: des intellectuels de premier plan (Vladimir Pečerin et Aleksandr Herzen par exemple) ont préféré quitter leur pays et se réfugier en Occident, plus libre et plus tolérant.

19 Le marquis de Custine pouvait s'appuyer sur des informateurs de premier choix. Sur le bateau qui l'amena de Travemünde à Kronstadt, il fit la connaissance du prince Pëtr B. Kozlovskij, un des rares catholiques de Russie, une figure pittoresque, diplomate, ami de Puškin, la langue bien pendue et comme chez lui dans tous les salons d'Europe occidentale. Il confia au marquis pas mal de réflexions amères sur la Russie, comme par exemple : «notre gouvernement vit de mensonge, car la vérité fait peur au tyran comme à l'esclave ». Au début, le Français refusait de croire son interlocuteur russe, mais, après avoir résidé un certain temps en Russie, il prit conscience de l'amère vérité de ses propos. Custine pouvait compter sur d'autres éminents Russes. En 1839, Aleksandr Turgenev fit savoir au prince Pëtr Vjazemskij que le marquis était en route pour la Russie et il demanda que son ami français le prît sous son aile et qu'il le recommandât auprès de Čaadaev, du prince Vladimir Odoevskij, etc. Custine avait donc pour informateurs des Russes parmi les plus intéressants, les plus éduqués et parlant très bien le français. Il fut également reçu à bras ouverts par Nicolas $\mathrm{I}^{\mathrm{er}}$ : il était connu pour ses opinions monarchistes et le gouvernement russe ne pouvait que mieux utiliser un occidental de premier plan qui ferait des commentaires positifs sur la Russie, laquelle avait mauvaise réputation en Occident, et particulièrement en France, surtout après la défaite de l'insurrection polonaise de 1830-1831. Avec la visite du marquis de Custine, le gouvernement russe espérait pêcher un gros poisson. Mais il en a été tout autrement. $L a$ Russie en 1839 exprimait une opinion cinglante sur l'autocratie russe. L'autocrate était furieux; il aurait alors déclaré: "C'est entièrement de ma faute, je n'aurais pas dû recevoir cette canaille. $»^{4}$ Mais l'autocrate russe n'a pas été le seul à être outré par le comportement ingrat de l'aristocrate français. Même certains esprits russes indépendants n'étaient pas contents des sévères critiques que Custine adressait à la Russie. Puškin n'a-til pas écrit à son ami Vjazemskij : «Bien sûr que je méprise ma patrie de la tête aux pieds, mais je suis contrarié quand un étranger partage mon sentiment... $»^{5}$. La russophobie doit donc être réservée aux Russes et rester entre eux, secrète (pas affichée en public, car il faut être loyal). Tjutčev fut indigné, Žukovskij trouvait que Custine méritait une gifle.

Le premier Russe qui réagit aux écrits du marquis de Custine fut Nikolaj Greč, lequel fit paraitre à Paris un livre dans lequel il tentait de placer La Russie en 1839 sous un jour moqueur: le livre serait plein de bêtises et donc pas fiable. Le critique Aleksandr Turgenev l'appelait « M-r Gretch, premier espion de sa majesté l'empereur de la Russie ${ }^{6}$. 
Mais la réfutation d'un Duez (« avocat à la cour royale de Paris ») a également paru sous le titre Critique des mystères de la Russie et de l'ouvrage de M. de Custine : "La Russie en 1839 », qui faisait valoir que dans la France de ces années-là de nombreux phénomènes avaient été bien plus tristes que ce que Custine avait vu en Russie. Jakov N. Tolstoj trouva nécessaire de lancer une attaque contre tous les publicistes anti-russes présentés dans la brochure Lettre d'un Russe à un journaliste français sur les diatribes de la presse anti-russe. Dans Un mot sur l'ouvrage de M. de Custine, Ksaverij Labenskij n'accusait pas le voyageur français d'avoir réuni des faits irréfutables, mais d'être trop négatif et trop général dans ses commentaires. La même année parut également Discours sur Pierre le Grand. Réfutation du livre de $M$. de Custine du comte Ivan Golovin, lequel travaillait au ministère russe des Affaires étrangères. Et enfin, je citerai l'opinion du slavophile Aleksej Homjakov (dans Moskvitjanin, en 1845) : il dit que de nombreux étrangers tiennent de Russes leur aversion pour la Russie. Les Russes en personne sont donc responsables du fait que sont publiés des livres négatifs dans ce genre.

21 Le marquis de Custine avait été si choqué par tout ce qu'il avait vu dans la Russie de Nicolas $\mathrm{I}^{\mathrm{er}}$ qu'en quittant le pays il appela à tous ses compatriotes :

Ce livre est remarquable non seulement parce qu'il dresse un tableau accablant de la Russie tsariste, mais également parce qu'il décrit la Russie communiste. Parmi les caractéristiques de l'autocratie notées par Custine, nombreuses sont celles que l'on retrouve sous Staline ou sous ses successeurs. Contre les critiques potentielles disant qu'il n'est resté que peu de temps ou qu'il ne connaissait pas la langue du peuple, Custine a prononcé ces mots célèbres : «J'ai mal vu, mais j'ai bien deviné. » Voici un livre qui, ayant donné comme une photographie de l'année 1839, a survécu au XIX ${ }^{\mathrm{e}}$ siècle et est devenu pendant la période du Rideau de fer un des livres les plus populaires et les plus en vue sur la Russie. On a usé (abusé) de ce livre pour imposer la thèse selon laquelle il n'y a pas vraiment eu de révolution en Russie, mais la tradition s'est prolongée (« en Russie cela a toujours été comme ça »). Ainsi, il est devenu un des livres influents de la Guerre froide. En Union soviétique, il a été publié une seule fois: en 1930. Ce n'est que pendant la perestroïka qu'il a été ressorti de l'ombre et depuis il a été édité à plusieurs reprises : en 1990 sous le titre Nikolaevskaja Rossija (La Russie de Nicolas), en 2000 sous la forme d'une édition critique Rossija $v 1839$ godu (La Russie en 1839). Finalement, ce livre influent a aussi trouvé sa place auprès du lecteur russe. Il est grand temps d'écrire un doctorat à ce sujet.

\section{NOTES}

1. En anglais dans le texte (NdT). Voir Rude and Barbarous Kingdom: Russia in the Accounts of Sixteenth-Century English voyagers (Un royaume violent et barbare: La Russie dans les récits des voyageurs anglais du XVI ${ }^{\mathrm{e}}$ siècle)(Sous la direction de Berry L. E. \& Crummey R. O.), University of Wisconsin Press, Madison, 1968.

2. Algarotti F., Viaggi di Russia (Voyage en Russie). Fondazione Bembo/Ugo Guanda, Parme, 1991, p. 55. 
3. Le livre d'Astolphe de Custine, La Russie en 1839 (Amyot, Paris, 1843) peut être consulté dans son entièreté sur le site : http://www.gutenberg.org/etext/25755.

4. Cf. l'histoire détaillée de la réception du livre de Custine dans: De Kjustin A., Nikolaevskaja Rossija (La Russie de Nicolas), AST, Moscou, 1990, pp. 3-47.

5. Puškin A. S., Lettre du 27 mai 1826, in:Sobranie sočinenij vdesjati tomah (đEuvres en dix volumes), t. IX, Hudožestvennaja literatura, Moscou, 1977, p. 219.

6. Ostaf'evskij arhiv (L'Archive d'Ostaf'ev), t. IV, Saint-Pétersbourg, 1899, p. 274.

INDEX

Index géographique : Russie

Index chronologique : XIXe siècle

oeuvretraite Custine (marquis de) A. : La Russie en 1839

\section{AUTEURS}

\section{EMMANUEL WAEGEMANS}

Professeur à l'Université Catholique de Leuven (KULeuven) 